\title{
Traceroute-based target link flooding attack detection scheme by analyzing hop count to the destination
}

\author{
Hiromu Asahina ${ }^{\text {a) }}$, Kei Sakuma, Shuichiro Haruta, \\ Hiroya Kato, and Iwao Sasase \\ Dept. of Information and Computer Science, Keio University, \\ 3-14-1 Hiyoshi, Kohoku, Yokohama, Kanagawa 223-8522, Japan \\ a)asahina@sasase.ics.keio.ac.jp
}

Abstract: In this paper, we propose traceroute-based target link flooding attack (TLFA) detection scheme by analyzing hop count to the destination. Since the destinations of the attacker's traceroutes tend to be concentrated around a target link, classifying traceroutes by hop count can highlight attacker's traceroute. Thus, the proposed scheme can detect the attack even if an attacker slowly executes traceroutes and the ratio of legitimate user's traceroutes is high. By computer simulations, we show our scheme has more robustness compared with the conventional scheme.

Keywords: DDoS attack detection, link flooding attack, security Classification: Internet

\section{References}

[1] M. S. Kang, S. B. Lee, and V. D. Gligor, "The crossfire attack," Proc. IEEE Symp. on Security and Privacy, pp. 127-141, May 2013. DOI:10.1109/SP. 2013.19

[2] H. Beitollahi and G. Deconinck, "Analyzing well-known countermeasures against distributed denial of service attacks," Comput. Commun., vol. 35, no. 11, pp. 1312-1332, 2012. DOI:10.1016/j.comcom.2012.04.008

[3] S. B. Lee, M. S. Kang, and V. D. Gligor, "CoDef: Collaborative defense against large-scale link-flooding attacks," Proc. ACM CoNEXT, pp. 417-428, Dec. 2013. DOI:10.1145/2535372.2535398

[4] L. Wang, Q. Li, Y. Jiang, and J. Wu, “Towards mitigating link flooding attack via incremental SDN deployment," Proc. IEEE Symposium on Computers and Communications, vol. 2016-August, pp. 397-402, 2016. DOI:10.1109/ISCC. 2016.7543772

[5] L. Xue, X. Luo, E. W. W. Chan, and X. Zhan, "Towards detecting target link flooding attack," Proc. USENIX LISA, Nov. 2014.

[6] T. Hirayama, K. Toyoda, and I. Sasase, "Fast target link flooding attack detection scheme by analyzing traceroute packets flow," Proc. WIFS, 2015. DOI:10.1109/ WIFS.2015.7368594

[7] J. Takeuchi and K. Yamanishi, "A unifying framework for detecting outliers and change points from time series," IEEE Trans. Knowl. Data Eng., vol. 18, no. 4, pp. 482-492, 2006. DOI:10.1109/TKDE.2006.1599387 
[8] A. Bradley, "The use of the area under the ROC curve in the evaluation of machine learning algorithms," Pattern Recognit., vol. 30, no. 7, pp. 1145-1159, 1997. DOI:10.1016/S0031-3203(96)00142-2

\section{Introduction}

Recently, target link flooding attack (TLFA) which is a new type of DDoS attack has been proposed [1]. This attack cuts off specific links, referred to as target links (TL), over the Internet and disconnects a specific region, referred to as a target area, from other regions. TLFA has two remarkable features: 1) the target area is not directly attacked and 2) the attacker uses valid IP addresses or transmits low rate traffic. For these reasons, conventional schemes against DDoS attacks [2] do not work effectively against TLFA. The attacker firstly selects the target area and decoy servers which are public ones located near the target area. Secondly, the attacker creates a map of network topology around the target area by employing a botnet and having bots send traceroute. Finally, the attacker selects bot-decoy pairs so that a path between the pair includes TL and commands bots to send an attacking flow. As a result, TLs are flooded and the target area is isolated.

In order to detect TLFA, several schemes have been proposed [3, 4, 5]. However, these schemes work after link congestion occurs. To deal with this shortcoming, Hirayama et al. [6] propose traceroute-based TLFA detection. Since an increase of traceroutes is an attack symptom, it is possible to detect the attack before the link congestion occurs by analyzing the number of traceroutes with a change point detection algorithm. However, this scheme cannot detect the attack when the ratio of legitimate user's traceroutes is high. This is because this scheme cannot distinguish a very gradual increase of attacker's traceroutes and a fluctuation of legitimate traceroutes. Unfortunately, the attacker can partially control the sending interval of traceroutes. Therefore, it is critical to detect the attack symptom from the small changes.

Thus, we propose traceroute-based TLFA detection scheme by analyzing hop count to the destination. Since the attacker needs to find routes to the public servers that contain TL, we argue that the destination of traceroute is concentrated within several hops from TL. Thus, by analyzing the number of traceroutes by hop counts, the change can be emphasized and the attack symptom might be more easily captured.

By computer simulations, we show our scheme has more robustness compared with the conventional scheme.

\section{Proposed scheme}

We propose traceroute-based TLFA detection scheme by analyzing hop count to the destination. We apply ChangeFinder [7], which is a change point detection algorithm, to time series data of traceroutes that are classified by hop count. This notion comes from the assumption that the number of attackers' traceroutes whose destination is within a few hops is much greater than that of legitimate traceroutes. Therefore, our scheme can emphasize the gradual increase of an attacker's tracer- 


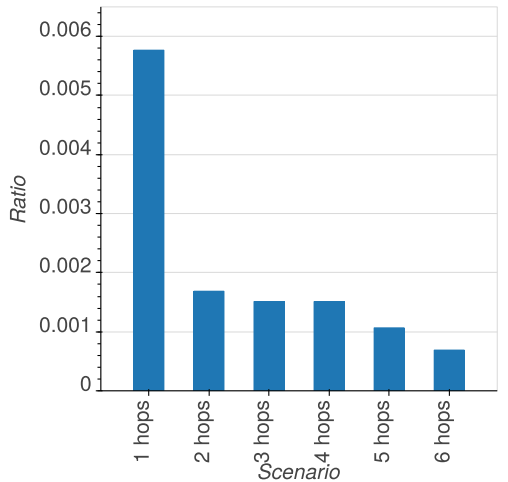

((a)) The ratio of paths including TL for each scenario.

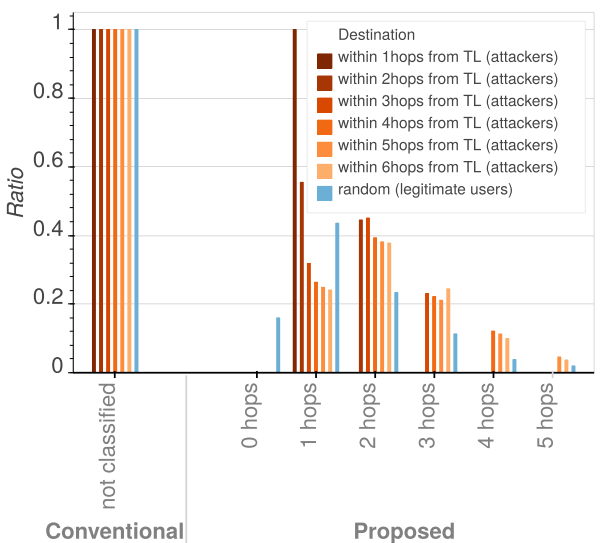

((b)) The ratio of changes observed by the proposed scheme when the changes observed by the conventional scheme always take 1 regardless of the scenarios.

Fig. 1. Simulation results for the prove of assumption.

outes and can easily capture the attack symptom. Our scheme is launched on a detection server in AS (Autonomous System). Since traceroute is multiple ICMP packets, the hop count can be identified from the TTL (Time To Live) field of these packets.

\subsection{Proof of the assumption}

In this section, the result of a simple simulation is presented in order to prove the assumption described in the previous section. We use the network topology consisting of 1,000 nodes $^{1}$. Our purpose here is clarifying percentage of paths including TL when choosing nodes $n$-hop away from TL as destinations. To realize this, we search the all shortest paths from the randomly chosen source to all destinations which are $n$-hop away from a randomly chosen TL and calculate the ratio of paths including TL. We consider six scenarios with different $n$ from 1 to 6 . This procedure is repeated until half of all nodes once become a source. Fig. 1(a) shows the ratio of paths including TL for each scenario. As shown in this figure, only $0.6 \%$ of paths include TL even if the destination is one hop away from TL. Based on this results, we argue that attackers have the motivation for choosing a node closer to TL as a destination.

Moreover, we simulate the situation where the conventional scheme cannot detect the attack to show the feasibility of the proposed scheme. According to the result of Fig. 1(a), we consider six types of attackers, i.e., attackers who randomly select a destination from nodes within 1, 2, 3, 4, 5 or 6 hops from TL. We also consider legitimate users who randomly select a destination from all nodes. Fig. 1(b) shows the amount of changes observed by the proposed scheme in the case where the amount of changes observed by the conventional scheme take 1. As shown in Fig. 1(b), all values labeled "attackers" exceed the value labeled "legitimate users" at either 1, 2, 3, 4 or 5 hops. This result indicates that the difference feasible for the detection can be observed by the proposed scheme.

${ }^{1}$ http://www.caida.org/data/as-relationships 


\subsection{Algorithm}

The detection servers collect a traceroute log. Traceroutes sent by legitimate users act as a 'noise'. After collecting traceroute logs, each detection server classifies them according to hop count. Let $m_{i j}$ denote the number of traceroutes whose destination is $j$ hops far from observing AS at time $i$. We consider $\boldsymbol{m}_{\boldsymbol{j}}=$ $\left(m_{1 j}, m_{2 j}, \ldots, m_{i j}, \ldots, m_{t j}\right)$. ChangeFinder first detects outliers in $\boldsymbol{m}_{\boldsymbol{j}}$ by calculating abnormality $a_{i j}$ from each point $m_{i j}$. Abnormality $a_{i j}$ is logarithmic loss score and calculated as

$$
a_{i j}=-\log p_{(i-1) j}\left(x=m_{i j} \mid m_{1 j}, \cdots, m_{(i-1) j}\right),
$$

where $p_{(i-1) j}(x)$ is the conditional probability density function calculated from $\left(m_{1 j}, \cdots, m_{(i-1) j}\right)$. This is defined as

$$
p_{(i-1) j}(x)=\frac{1}{\sqrt{2 \pi} \sigma} \exp \left(-\frac{(x-w)^{2}}{2 \sigma}\right),
$$

where $\sigma$ denotes a variance of a noise term $\epsilon$ in the $k$-order AR model and $w$ is denoted as $w=\sum_{n}^{k} \alpha_{n}\left(m_{i-n}-\epsilon\right)-\epsilon$. Then, in order to avoid the situation where single outlier causes false attack detection, we apply smoothing processing with window size $\mathrm{W}$ to $a_{i j}$ for $n=[W, t]$, where $t$ denote the current time. The smoothing processing is calculated as

$$
\bar{a}_{i j}=\frac{1}{W} \sum_{n=i-W+1}^{i} a_{n j} .
$$

The final score $s_{i j}$ is calculated as

$$
s_{i j}=-\log p_{(i-1) j}\left(x=\bar{a}_{i j} \mid \bar{a}_{1 j}, \cdots, \bar{a}_{(i-1) j}\right),
$$

where $s_{i j}$ denote the final score of $m_{i j}$. Finally, let $d_{t h}$ be the predefined threshold value. For $i=[2, t]$, detection servers judge the change as an attack if the condition $s_{i j}-s_{(i-1) j}>d_{t h}$ holds. In an actual environment, $d_{t h}$ can be configured based on a legitimate traceroutes $\log$ on the detection server so that the change caused by legitimate traceroutes is not detected. The above procedures are executed for all $j$.

\section{Evaluation}

We evaluate our scheme by computer simulation. To show effectiveness of our scheme, we introduce AUC (Area Under the ROC Curve) [8]. In order to calculate AUC, each detection server calculates ROC curves with different thresholds $d_{t h}=(0.0,0.1, \ldots, 20.0)$. If an algorithm perfectly classifies attacks and nonattacks, the AUC indicates 1.0.

\subsection{Simulation setup}

We compare the detection accuracy of the proposed scheme with that of the conventional scheme from two perspectives: (i) the amount of noise versus the number of ASes which achieve high AUC; and (ii) AUC while changing the sending interval of attacker's traceroutes. 


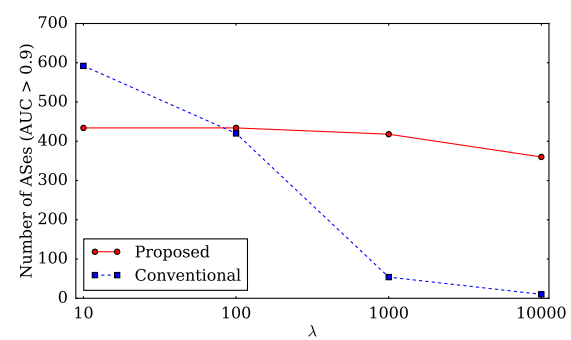

((a)) Number of ASes vs noise.

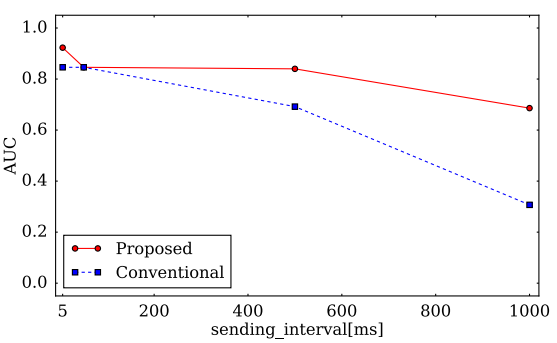

((b)) AUC vs sending interval.

Fig. 2. Simulation results.

We construct network topology using Caida AS Relationships Dataset ${ }^{2}$. We assume that each AS has only one router to simplify simulations. In accordance with [1], the number of bots and decoy servers is set to twice as many as the number of ASes. A bot sends traceroutes to all decoy servers three times at any given point in time. On the other hand, a legitimate user sends traceroute to a randomly chosen destination. The number of traceroutes on each AS follows the Poisson distribution with $\lambda$.

\subsection{Simulation methodology}

In order to find the abnormal point, we generate two time series data as follows: 1) Training data: A time series data which includes only the number of traceroutes sent by legitimate users. 2) Test data: A time series data which includes the number of traceroutes sent by bots and legitimate users. Firstly, each detection server loads training data, and then judges a given test data with threshold $d_{t h}$. Here, the time interval in Eq. (1)-(4) is 5 millisecond.

\subsection{Simulation results}

Fig. 2(a) shows the number of ASes that achieve AUC $>0.9$ versus noise. As shown in Fig. 2(a), the conventional scheme cannot detect the attack when $\lambda$ is larger than 1,000. On the contrary, in the proposed scheme, a lot of ASes can detect the attack. This is because the effect of the noise in the proposed scheme is less than that of the conventional scheme since the noise is distributed among multiple hop counts. However, when the noise is 10, the number of ASes with the proposed scheme which achieve higher AUC is smaller than the conventional scheme. This is because attacker's traceroutes are divided into several hops and the change cannot be detected in each hop count. However, since both proposed and conventional schemes can be used complementarily, it is not a critical problem. Furthermore, according to a packet capture dataset ${ }^{3}$ in Dutch Academic, traceroute is generated 5 packets per second in each global IP address. It is unrealistic situation that there are less than 100 legitimate user's traceroutes per second in each AS.

Fig. 2(b) shows AUC versus traceroute sending interval. As can be seen from Fig. 2(b), AUC of the conventional scheme decreases as the sending interval increases. On the contrary, the proposed scheme keeps higher AUC than the conventional scheme in all cases. It is easily guessed that the sufficient detection

\footnotetext{
${ }^{2}$ http://www.caida.org/data/as-relationships

${ }^{3}$ https://traces.simpleweb.org/traces/TCP-IP/location3/
} 
accuracy cannot be maintained if the sending interval is further lengthened. However, the attacker cannot unduly lengthen the sending interval because more than $30 \%$ of network topology is dynamically changed [1].

\section{Conclusion}

In this paper, we have proposed traceroute-based TLFA detection scheme by analyzing hop count to the destination. The proposed scheme successfully overcomes the shortcoming of the conventional scheme. The performance evaluation results show that our scheme can detect the attack symptom when noise and traceroute sending interval increase.

\section{Acknowledgments}

This work is partly supported by the Grant in Aid for Scientific Research (No. 17K06440) from Japan Society for Promotion of Science (JSPS). 